\title{
NUMERICAL STUDY OF STRESS-STRAIN LOCALIZATION IN THE TITANIUM SURFACE MODIFIED BY AN ELECTRON BEAM TREATMENT
}

\author{
UDC 531.3
}

\author{
Ruslan Balokhonov ${ }^{1,2}$, Varvara Romanova ${ }^{1}$, Alexey Panin ${ }^{1}$, \\ Sergey Martynov ${ }^{1}$, Marina Kazachenok ${ }^{1}$ \\ ${ }^{1}$ Institute of Strength Physics and Materials Science, Tomsk, Russian Federation \\ ${ }^{2}$ National Research Tomsk Polytechnic University, Tomsk, Russian Federation
}

\begin{abstract}
Numerical simulation is performed to investigate the mesoscale stress-strain localization in a surface-modified commercial titanium alloy. The calculated crystalline microstructure corresponds to that observed in experiments and is accounted for in an explicit way as initial conditions of a dynamic boundary-value problem. The latter is stated in terms of plane strain developing in microstructure subjected to tension and is solved numerically by the finite-difference method. Elastic-plastic constitutive models were built to describe the experimental mechanical response both of the substrate and of the modified layer. Plastic strain localization is found to depend on the grain yield strength.
\end{abstract}

Key Words: Microstructure-based Numerical Simulation, Plastic Strain Localization, Titanium Alloys, Surface-modified Materials

\section{INTRODUCTION}

Stress-strain localization phenomena have been much studied both experimentally and theoretically (see, for instance, a review [1]) and may be due to different physical processes operating at different scale levels. At the microlevel, dislocations, slip bands, dislocation cells, fragmented structures, etc., are formed in single crystals and in grains of polycrystals. The macroscopic stress-strain localization may be due to the geometry of structure elements or specimens, or else, to loading conditions. In simulating localization effects observed in homogeneous specimens under loading, the form of the plastic

Received October 10, 2016 / Accepted November 21, 2016

Corresponding author: Ruslan Balokhonov

Institute of Strength Physics and Materials Science, Russian Academy of Sciences, 2/4, pr. Akademicheskii,

634055 Tomsk, Russia

E-mail: rusy@ispms.tsc.ru 
potential [2] generally plays a decisive role. The softening rate was a decisive factor for the onset of localization [3]. To describe the final fracture in the form of strain localization in a polycrystalline material, there was developed a crystal plasticity model with a hardening law accounting for slip and twin systems [4]. It was shown in [5, 6] that a large deformation gradient theory can provide a localized shear band thickness in the neck, no matter what the computational grid size is. A non-local constitutive formulation including a gradient internal length of poroplastic materials was given in [7] to derive analytical expressions of the critical hardening/softening modulus for localized failure.

Since the mid-1980s the mesoscopic stress-strain localization associated with different interfaces were studied numerically in many papers where an explicit account is taken of the material microstructure (see, e.g. [8-12]. In our earlier works we investigated stress-strain localization processes in polycrystalline materials [13, 14], metal-matrix composites [15, 16] and materials with coatings $[17,18]$. It has been shown numerically that the curvature of the coating-substrate interface is responsible for the stress concentration that gives rise to plastic strain localization in metal substrates and causes fracture of ceramic coatings. The level of local stresses near an interface of certain curvature depends on the difference in the mechanical properties between the contacting materials at the interface. An explicit account of microstructure of coated titanium was not considered in the literature yet. The aim of the present work is to simulate numerically the evolution of the mesoscale stress concentration and plastic strain localization in surface modified commercial VT1-0 titanium samples subjected to tension, with the respective polycrystalline and submicrocrystalline structures of the titanium substrate and modified surface layer being taken into account.

\section{FoRMULATION OF THE PROBLEM AND EXPERIMENT}

Use was made of low-energy high-current electron beam (LEHCEB) treatment to provide a combination of melting and fast crystallization of a thin surface layer of VT1-0 titanium samples. The crystalline microstructure and modified surface layer thickness were found to depend on the energy density, with a curvilinear interface being formed between the substrate and the modified surface layer (Fig. 1a) [19].

The experiments under consideration included the following stages. Dumb-bell test pieces with a gage section of $2 \times 1 \times 12 \mathrm{~mm}$ were cut out by wire electrical discharge machining. The test pieces were annealed in a vacuum chamber at a temperature of $750^{\circ} \mathrm{C}$ for $1 \mathrm{~h}$ and polished mechanically. Uniaxial tension of the titanium samples was performed in an INSTRON 5582 tensile testing machine at room temperature and a loading rate of $0.3 \mathrm{~mm} / \mathrm{min}$. The stress-strain behavior is shown by dots in Fig. 1b. The flat surfaces of the samples were exposed to three 50 $\mu$ s pulses using a SOLO electron beam system (Institute of High Current Electronics SB RAS, Tomsk, Russia). The beam energy density was $18 \mathrm{~J} / \mathrm{cm}^{2}$ and the pulse repetition frequency was $0.3 \mathrm{~Hz}$. Irradiation was performed in argon atmosphere with a residual pressure of $0.02 \mathrm{~Pa}$. A JEM-2100 transmission electron microscope (TEM) was employed to examine the microstructure of the samples before and after the electron beam processing. The hardness and elastic moduli of the titanium substrate and modified surface layer were measured with a NanoTest nanotester equipped with Berkovich pyramid.

The calculations relied on an explicit account of the experimental microstructure (Fig. $1 \mathrm{c}-\mathrm{d})$. The associated tension tests were simulated. The dynamic boundary-value problem 
represented in the plane strain formulation was solved numerically by the finite-difference method [17]. Elastic-plastic constitutive models were built to describe the experimental mechanical response both of the substrate and of the surface layer. The isotropic strain hardening function confines the equivalent stress:

$$
\sigma_{e q}=\sigma_{s}-\left(\sigma_{s}-\sigma_{0}\right)^{-\varepsilon_{e q}^{p} / \varepsilon_{r}^{p}},
$$

where $\boldsymbol{\varepsilon}_{e q}^{p}$ is the equivalent plastic strain, $\sigma_{s}$ and $\sigma_{0}$ are the ultimate stress and yield stress, and $\boldsymbol{\varepsilon}_{r}^{p}$ is a representative value of the equivalent plastic strain. To allow for the stochastic orientation of crystallites in the modified layer relative to the loading direction, the yield stress, elastic shear, and bulk moduli were varied in a random way from one crystallite to the other within the ranges presented in Table 1 . The average yield strength of the crystallites was calculated as the substrate yield strength multiplied by the ratio of surface layer-to-substrate hardness. The experimental ratio was found to be 1.25, whereas the ratio of surface layer-tosubstrate elastic moduli was 1.1. That is why different combinations of three values of the yield strength and shear $(\mu)$ and bulk $(\mathrm{K})$ moduli were used for the substrate grains (see Table 1 ). The strain hardening function for grains characterized by average properties accounts for the experimental stress-strain behavior of the titanium substrate (Fig. 1b).

Table 1 Mechanical properties of grains and crystallites used in the calculations

\begin{tabular}{lccccc}
\hline & $\sigma_{0}[\mathrm{MPa}]$ & $\sigma_{s}[\mathrm{MPa}]$ & $\varepsilon_{\mathrm{r}}^{\mathrm{p}}[\%]$ & $\mathrm{K}[\mathrm{GPa}]$ & $\mu[\mathrm{GPa}]$ \\
\hline Crystallites & $113-338$ & 388 & 0.13 & $73-102$ & $52-74$ \\
Grains & $90,180,270$ & 310 & 0.13 & $66,79,93$ & $47,57,67$ \\
\hline
\end{tabular}

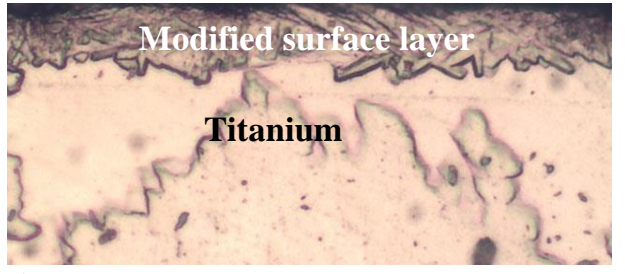

a)

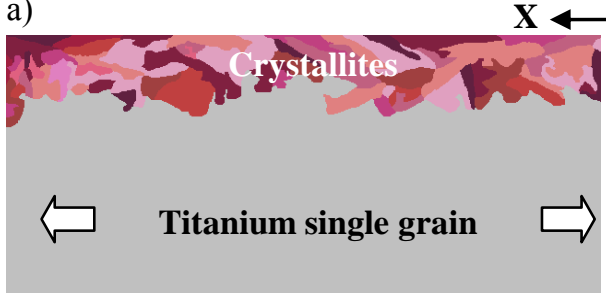

(c)

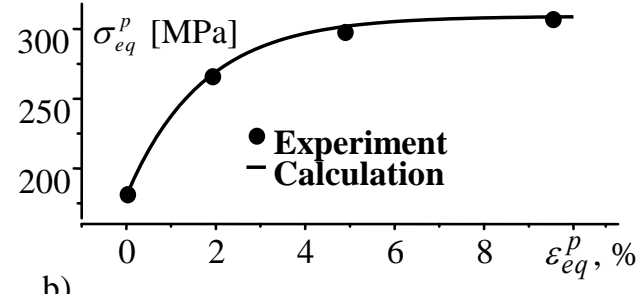

b)

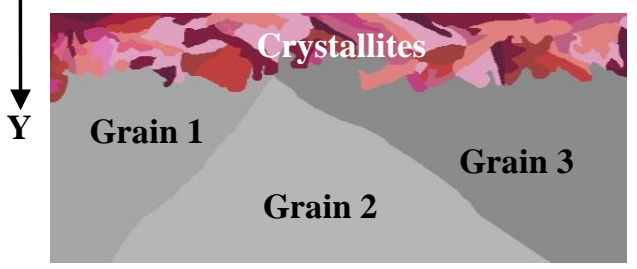

(d)

Fig. 1 Experimental (a) and simulated microstructures (b-c) of a commercial VT1-0 titanium substrate with a modified surface layer produced by means of LEHCEB surface irradiation and a plastic potential for a titanium sample (d) 
The boundary conditions for the top and bottom surfaces correspond to a free surface and symmetry plane, respectively. The right- and left-hand surfaces simulate uniaxial elongation of the polycrystalline microstructures along the $X$-axis.

\section{Plastic Strain Localization at the Mesoscale}

Two sets of calculations were performed. One set deals with the elongation of a single titanium grain with a modified surface layer (Fig. 1c). The yield strength of the substrate grain was 90, 180, and $270 \mathrm{MPa}$ (Table 1). The plastic strains were found to originate near the curvilinear interface between the substrate and the modified surface layer, giving rise to a system of slip bands that develop in conjugated directions and compensate for the local bending of the material (Figs. $2 \mathrm{a}$ and $2 \mathrm{~b}$ ). In a qualitative sense, the same strain patterns are observed in the experiments (Fig. 2c). The plastic strain localization is shown to be more pronounced in grains possessing higher resolved shear stress and stiffness than in those favorably oriented in the loading direction (Figs. 2a and 2b).

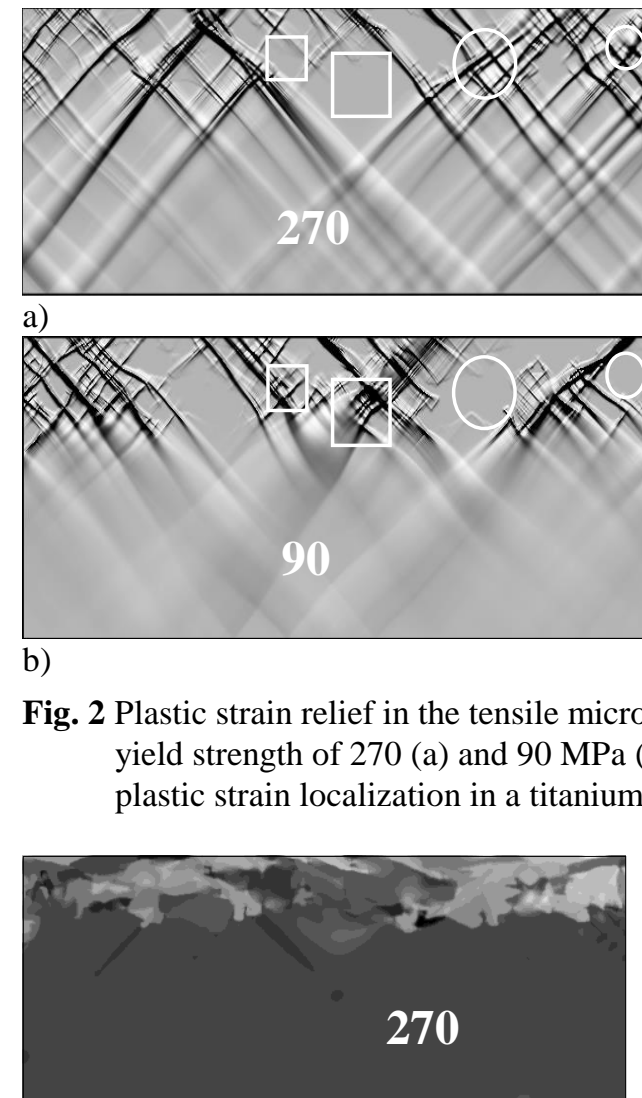

b)

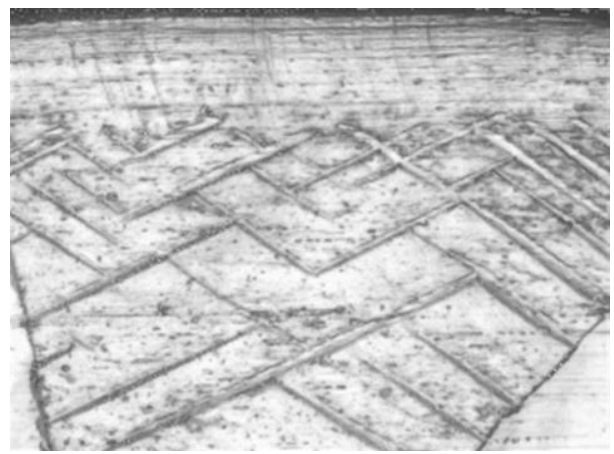

c)
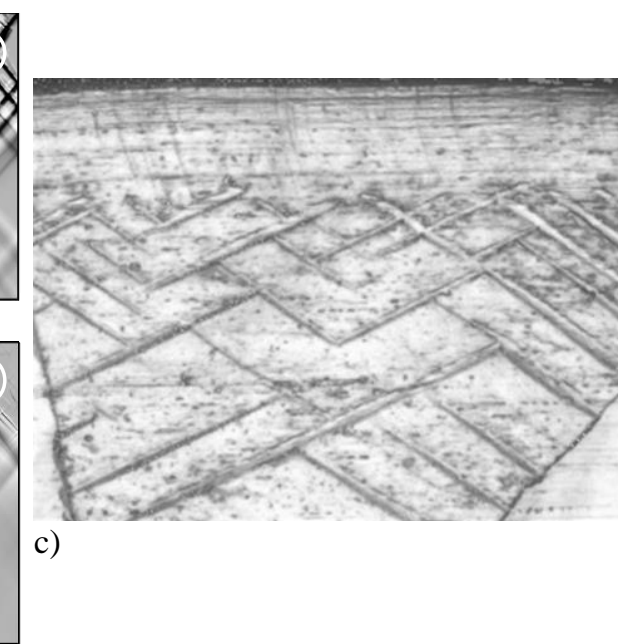

microstructure presented in Fig. 1c with substrate yield strength of 270 (a) and $90 \mathrm{MPa}$ (b) and an experimental TEM image of the plastic strain localization in a titanium grain with a modified surface layer (c)

a)

Fig. 3 Equivalent stress patterns for the cases presented in Fig. $2 \mathrm{a}$ and $\mathrm{b}$

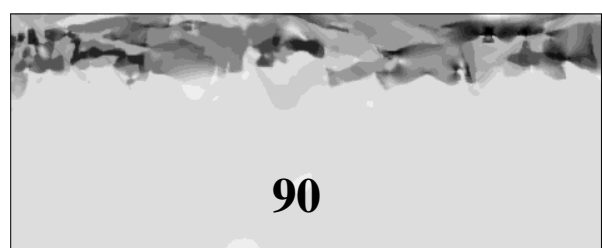

b) 
Note that the only difference between the cases presented in Figs. $2 a$ and $2 b$ is the magnitude of the yield stress of the substrate grain, with all the other factors, among which are the microstructure and distribution of the mechanical properties of crystallites in the modified surface layer, being the same. Despite this, the plastic strain patterns in the modified layer differ widely between the two cases at hand. For instance, there are crystallites and crystallite groups that experience large strains in one case or undergo no plastic flow at all in the other case (circles in Figs. $2 a$ and $b$ ), and vice versa (squares in Figs. $2 \mathrm{a}$ and $\mathrm{b}$ ). This is due to the difference in the plastic strain localization histories between the titanium substrate grains lying below the modified layer. For a titanium grain unfavorably oriented relative to the loading direction, the modified surface layer is exposed to low stresses (Fig. 3a). Plastic flow originates in the surface layer. On further loading the plastic strain localization pattern formed in the surface layer controls the plastic strain generation and localization in the substrate grain. Other scenario is realized in the substrate grain possessing low yield stress, where the microcrystalline layer formed on the grain surface is hardened (Fig. 3b). In this case, plastic flow originates in the grains lying near the interface between the substrate and the modified surface layer, and the plastic strain localization pattern in the grains depends on the degree of interfacial curvature.

The other set of calculations is devoted to a simulation of deformation of the microstructure containing three grains in the substrate (Fig. 4). The highest stress concentration and plastic strain localization are observed in a triple (hard-soft-hard grains) junction, and the plastic strain localization bands formed in hard grains are curved due to grain rotation caused by plastic straining of a soft grain lying in between (Fig. 4a).
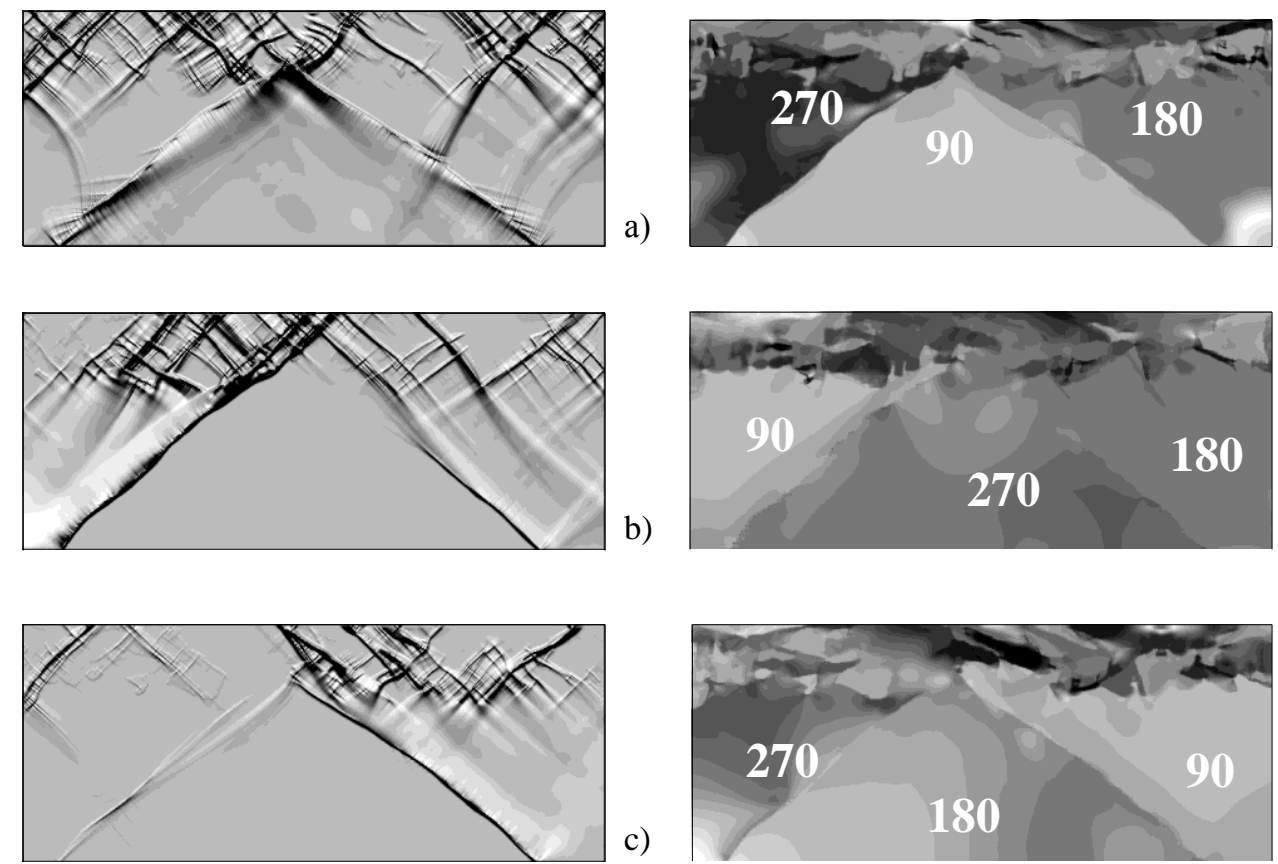

Fig. 4 Plastic strain relief and stress patterns in the tensile microstructure given in Fig. 1d 


\section{CONCLUSION}

Stress-strain localization in surface modified titanium subjected to tension is investigated numerically. Plastic strain patterns in a microcrystalline modified surface layer lying over titanium grains of different orientations relative to the loading direction are found to be dramatically different. Plastic strain localization is most pronounced in titanium grains undergoing high resolved shear stresses, and the plastic strain localization bands assume a curvilinear form due to grain rotation.

Acknowledgements: The work is supported by Russian Science Foundation (Project No. 14-19-00766).

\section{REFERENCES}

1. Antolovich, S.D., Armstrong, R.W., 2014, Plastic strain localization in metals: origins and consequences, Progress in Materials Science, 59, pp. 1-160.

2. Becker, R., Needleman, A., 1986, Effect of yield surface curvature on necking and failure in porous plastic solids, J. Appl. Mech., 53, pp. 491-499.

3. Xue, L., 2010, Localization conditions and diffused necking for damage plastic solids, Engineering Fracture Mechanics, 77, pp. 1275-1297.

4. Wang, Y.Y., Sun, X., Wang, Y.D., Hu, X.H., Zbib, H.M., 2014, A mechanism-based model for deformation twinning in polycrystalline FCC steel, Materials Science \& Engineering A, 607, pp. 206-218.

5. Borg, U., 2007, Strain gradient crystal plasticity effects on flow localization. International Journal of Plasticity, 23, pp. 1400-1416

6. Anand, L., Aslan, O., Chester, S.A., 2012, A large-deformation gradient theory for elastic-plastic materials: Strain softening and regularization of shear bands, International Journal of Plasticity, 30-31, pp. 116-143.

7. Mroginski J.L., Guillermo E., 2014, Discontinuous bifurcation analysis of thermodynamically consistent gradient poroplastic materials, International Journal of Solids and Structures, 51, pp. 1834-1846

8. Llorca, J., Needleman, A., Suresh, S., 1991, An analysis of the effects of matrix void growth on deformation and ductility in metal-ceramic composites, Acta Metallurgica et Materialia, 39, pp. 2317-2335.

9. Needleman, A., 2000, Computational mechanics at the mesoscale. Acta Materialia, 48, pp. 105-124.

10. Panin, V.E., (Eds.), 1998, Physical mesomechanics of heterogeneous media and computer-aided design of materials, Cambridge International Science Publishing, Cambridge, $380 \mathrm{pp}$.

11. Diard, O., Leclercq, S., Rousselier, G., and Cailletaud, G., 2005, Evaluation of finite element based analysis of $3 D$ multicrystalline aggregates plasticity: Application to crystal plasticity model identification and the study of stress and strain fields near grain boundaries, International Journal of Plasticity, 21, pp. 691-722.

12. Williams, J.J., Segurado, J., LLorca, J., Chawla, N., 2012, Three dimensional (3D) microstructure-based modeling of interfacial decohesion in particle reinforced metal matrix composites, Materials Science \& Engineering A, 557, pp. 113-118.

13. Romanova V.A., Balokhonov R.R., 2009, Numerical simulation of surface and bulk deformation in three-dimensional polycrystals, Physical Mesomechanics, 12, 3-4, pp. 130-140.

14. Balokhonov R.R., Romanova V.A., Batukhtina E.E., Martynov S.A., Zinovjev A.V., Zinovjeva O.S., 2016, A mesomechanical analysis of the stress-strain localisation in friction stir welds of polycrystalline aluminum alloys, Meccanica, 51 (2), pp. 319-328.

15. Romanova V., Balokhonov R., Makarov P., Schmauder S. and Soppa E., 2003, Simulation of elastoplastic behaviour of an artificial 3D-structure under dynamic loading, Computational Materials Science, 28 (3-4), pp. 518-528.

16. Balokhonov R.R., Romanova V.A., Schmauder S., 2006, Computational analysis of deformation and fracture in a composite material on the mesoscale level, Computational Materials Science, 37, pp. 110-118.

17. Balokhonov, R.R., Romanova, V.A., 2009, The effect of the irregular interface geometry in deformation and fracture of a steel substrate-boride coating composite, International Journal of Plasticity, 25 (11), pp. 2225-2248.

18. Balokhonov R.R., Zinovjev A.V., Romanova V.A., Bakeev R.A, Zinovjeva O.S., 2016, Numerical simulation of deformation and fracture in a material with a polysilazane-based coating, Physical Mesomechanics, 19 (4), pp. 430-440.

19. Panin A.V., Kazachenok M.S., Kretova O.M., Perevalova O.B., Ivanov Y.F., Lider A.M., Stepanova O.M., and Kroening M.H., 2013, The effect of electron beam treatment on hydrogen sorption ability of commercially pure titanium, Appl. Surf. Scie., 284, pp. 750-756. 